\title{
A Low-Delay and Robust Solution for Rapid Movement Detection Using UWB
}

\author{
Z. Y. Liu ${ }^{1}$, R. Z. Chen ${ }^{1}$ *, F. Ye ${ }^{1}$, G. Y. Guo ${ }^{1}$, L. X. Huang ${ }^{1}$ \\ ${ }^{1}$ State Key Laboratory of Information Engineering in Surveying, Mapping and Remote Sensing \\ (LIESMARS), Wuhan University, Wuhan 430079, China
}

KEY WORDS: Low delay, Rapid movement, UWB, C-TWR, NLOS, TDMA, A-EKF

\begin{abstract}
:
The increasing applications for location-based services (LBS) and real-time location system (RTLS) promote the development of indoor positioning and navigation technology. However, the positioning methods based on traditional Radio Frequency (RF) signals are difficult to improve the accuracy and sensitivity for rapid movement detection with a low latency parameter. In order to solve those problems, this paper adopted ultra-wideband (UWB) chips as the wireless communication technology and designed a method of rapid movement detection with low delay. The method consists of a topology of combined two way ranging (C-TWR), a schedule of time division multiple access (TDMA), an non-light-of-sight (NLOS) handling mechanism and an adaptive extended Kalman filter (A-EKF). To examine the low delay performance, accuracy and robustness of the system, a specific electro-displacement actuator with programmable speed is applied to provide precise ground truth for real speed and distance, and a system test platform consisting of four anchors and four tags provides information of specific situation. The results show that the system can locate the rapid moving pedestrian with a maximal speed of approximately $5 \mathrm{~m} \cdot \mathrm{s}^{-1}$ in real-time with a positioning accuracy of less than $0.5 \mathrm{~m}$. There are profound implications for further studies in the range of human wearable tags.
\end{abstract}

\section{INTRODUCTION}

Over the past decades, an increasing number of researchers, engineers, systems designers and amateurs have been involved in the research of indoor localization and navigation (Koyuncu, Yang, 2011). There are massive studies about indoor positioning technologies including geomagnetic fields, Wi-Fi, bluetooth low energy (Hammeed, Ahmed, 2018) and visual reality, which promise to satisfy the demand for the real-time localization systems (RTLS) in a wide range of scenarios such as emergency rescue, automatic navigation, material management, and motion detection (Risset et al., 2018), etc.

The applications of rapid movement detection are drawing more attention with spawning new research hotspot and market trend, especially in sports training (Leser et al., 2011, Ridolfi et al., 2018): track detection for runners, speed recording for bikers, and wearable solutions in other sports (Banerjee, et al., 2017) etc. The techniques applied in these systems mostly aim at measuring distance and evaluating angle or depth inversion. The performance of these systems including precision and reliability is highly impacted by the precision of the ranging, the resolution of the angle, algorithm complexity and the real-time. However, the traditional Radio Frequency (RF) (Vorst et al., 2008) based on indoor positioning methods such as received signal-strength (RSS) and angle of arrival (AOA) (Guolin et al., 2005), are difficult to improve the accuracy and sensitivity of these systems. Motion capture system (Mocap) technologies, with optical, image or hybrid systems, are featured with high cost, poor portability, small scope, omnidirectional camera and massive calculation (Joo et al., 2015), and mainly design for specific purposes. Although inertial measurement units (IMUs) are suitable and wearable, yet the accumulated error limits the long-term operation (Pham, Suh, 2016). Roundtrip time-offlight (RToF) based (Nuaimi, Kamel, 2011) methods are commonly considered as the best candidate for precise and stability measurements. This study aims to uncover the method of rapid movement detection applications in sports training with low delay by using the accurate RToF technology.

Here, ultra-wideband (UWB) acts as the representative of RToF fundamental approaches (Ding et al., 2010), providing far more instant and precise ranging performance than other positioning technology. It also presents a low delay measurement capability due to its impulse radio scheme large bandwidth, low power spectral density, and fine time resolution. UWB transceiver is always considered as one of the most suitable approaches for rapid movement systems in which the move always characterized by fast changes in direction and velocity. Although this technology has been applied for a long time in industry, yet only in recently years it has been integrated into many civilian applications. The DW1000 designed by Decawave (Decawave, 2018) is a single powerful chip compatible with the IEEE 802.15.4-2011 standard (IEEE Standard for low-rate wireless networks, 2016) and has already been integrated into many commercial systems.

In the IEEE802.15.4a standard, the familiar ranging localization technology with UWB is presented, which was named by symmetrical double-side two way ranging (SDS-TWR). To get more reliable ranging measurements, information handshaking should increase by three or even four times in this mechanism. This method is often poor in real-time, and can be further improved with a combined TWR (C-TWR) scheme. In the CTWR topology, the channel occupancy time can be optimized to improve the real-time of ranging. Due to the clock drift of each nodes' crystal oscillator, the carrier integrator value provided by DW1000 chips is used to calculate the clock offset ratio, to correct the different local and remote clock rates and reduce random errors. Furthermore, in order to guarantee the user densities and overcome multi-user interference of existing approaches, this study implements a time division multiple

* Corresponding author 
access (TDMA) for each rover node, which effectively avoids the large channel occupancy in the process of ranging and positioning.

The paper contains five sections as follows. Section 2 shows an overview of the ranging scheme of C-TWR, TDMA technology, some details of clock correction model, NLOS diagnose and localization algorithms. Section 3 describes the experimental setup of different scenarios, including low delay ranging and dynamic positioning. Meanwhile, the results are presented in Section 4 and the conclusions are illustrated in Section 5.

\section{METHODS}

\subsection{Ranging scheme}

The UWB based positioning system consists of wireless network nodes, including initiators and responders, where the ranging is measured with the assistance of time of flight (TOF) technology. Considering the clock drift of each nodes involving in the wireless networks, strict timing constrains should be comprehensively considered. TWR-based approach is a common way for symmetric double side two way ranging (SDSTWR), which eliminates the ranging error arising out of the local clock drift of individual participants through symmetric response time. Nevertheless, this scheme requires more than three times of information handshaking to accomplish one sample ranging between one tag and one anchor. Since serious expenditure of time the topology is highly precise, it is frequently used in science and industry (Silva et al., 2014). Furthermore, due to that it always occupies large channel with the number of anchors increasing, the topology is hardly to satisfy the operation of multi-user.

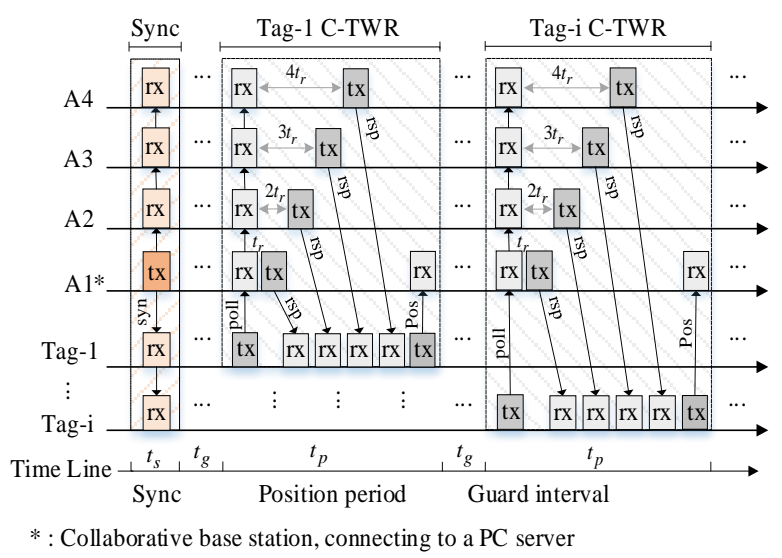

Figure 1. The schematic diagram of the positioning schedule.

To lower the channel usage and improve the efficiency of ranging, the C-TWR based on the traditional topology is employed, which utilizes the parallel receiving capabilities of the anchor nodes. The details are illustrated in Fig. 1. In this asymmetric ranging scheme, the anchor stations reply the poll packet issued by a tag station in various pre-defined time intervals, which effectively decreases the total number of packets with position estimation. It improves the real-time capabilities and optimizes the energy efficiency. The distances between the tag and the anchors can be measured even based on one poll message from the tag since all the anchors would receive it and response. Then the tag station can realize selfpositioning with more than two measurements. The final measurement results are sent to the collaborative base station in a communicate message, collected and displayed by a PC server. To increase the user density and the update dates of ranging, the ranging scheme is further improved with a TDMA schedule. The overall design of the schematic diagram is depicted in Fig. 1. A larger percentage of air time can be used in the scheduler because the system includes coordinating mechanisms to control when nodes transmit.

In details, a periodic wireless synchronization message initiated by a coordinating node is designed to manage the communication time sequence of the system. And the message would clear the time counter for all the mobile station and then uniform the time-line, similar to global navigation satellite systems (GNSS) operation. The period of the synchronization message is set to $5 \mathrm{~s}$. Each individual tag station synchronises with the message and transmits poll packet only in the assigned time slot. Meanwhile, all clocks of the fixed anchors are also aligned to the common reference time-line.

For multi-user applications, the positioning period of a tag, known as update rate, can be adjusted dynamically in according with the number of users and the time consumption of one ranging. From Fig.1, we can get the correlation between the update rate of ranging and user density. It can be described as

$$
f_{\text {update }}=\frac{1}{\left(t_{p}+t_{g}\right) \times N}
$$

where

$$
\begin{aligned}
& f_{\text {update }}=\text { update rate for one rover } \\
& t_{p}=\text { time of one simple positioning period } \\
& t_{g}=\text { guard interval between two C-TWR ranging } \\
& N=\text { number of users }
\end{aligned}
$$

\subsection{Clock correction model}

In practical system, due to the clock drift of each nodes' crystal oscillator, all the base stations and the mobile stations are difficultly to keep the same clock. Generally, a common integrated oscillator can be calibrated to up $\pm 2 \mathrm{ppm}$ (Decawave, 2017). Even so, the error of a round-trip time measurement is also scabrous for many scenarios.

To describe the effect of the oscillator performance on TWR ranging, we can calculate the error in one time of flight measurement according to the following equation

$$
t_{e}=\frac{t_{r p} \cdot \Delta \varepsilon}{2}
$$

where $t_{r p}=$ reply time of one ranging,

$$
\Delta \varepsilon=\text { relative clock drift between two transceivers }
$$

According to (2), an error of up to $4 \mathrm{~ns}$ can appear with a relative clock drift of $\pm 2 \mathrm{ppm}$ and a reply time of $1 \mathrm{~ms}$. And it corresponds to a distance of $1.2 \mathrm{~m}$, which is almost lethal for many applications.

Due to the asymmetric reply time, C-TWR based topology cannot eliminate the local clock drift error, unlike SDS-TWR based approach. Fortunately, the DW1000 chips designed by Decawave provides carrier integrator value to compensate the clock drift between the local and remote parts by calculating the 
clock offset ratio. The magic operation effectively reduces random error rate for a simple TWR ranging. When a message packet is successfully received, the carrier integrator register (CIR) of DW1000 can be read and convert to the absolute frequency error (in ppm) by using

$$
\text { offest }_{p p m}=-10^{6} \times \frac{C \times F_{s} \times 2^{-17}}{2 \times N_{s} \times F_{c}}
$$

where

$C=$ carrier integrator value

$N_{s}=$ the number of CIR sampling points

$F_{s}=$ the sampling frequency of CIR

$F_{c}=6489.6 \mathrm{MHz}$ for channel 5 , representative

The value of $N_{s}$ and $F_{s}$ are both constant.

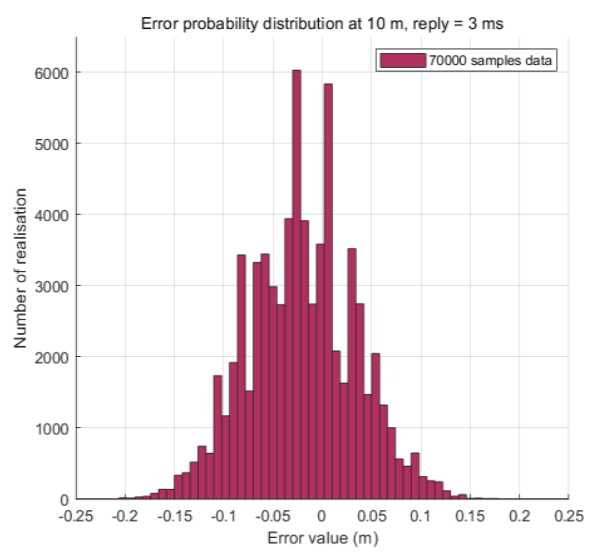

Figure 2. Error distribution on static ranging at $10 \mathrm{~m}$ apart between tag and anchor (reply $=3 \mathrm{~ms}$ ).

An experimental result of error distribution on static ranging is shown in Fig. 2. The experimental is done with a relative clock drift of $\pm 2 \mathrm{ppm}$, a reply time of $3 \mathrm{~ms}$ and $10 \mathrm{~m}$ apart between the tag and anchor. The measurement results indicate that an error of approximately $\pm 15 \mathrm{~cm}$ can appear where the time reply is set as $3 \mathrm{~ms}$.

\subsection{NLOS diagnose}

For wireless networks, operation in NLOS situations may only result in signal level attenuation, but not loss of data such as Wi-Fi and Bluetooth. However, for UWB systems where the objective is measuring distance, NLOS may result in a serious ranging error. Therefore, a non-light-of-sight handling mechanism must be considered in practical applications, especially in the scenario that the signal occlusion and object shelter are serious.

The NLOS situation may happen at any moment when the pedestrian is moving dynamically. In this paper, we use the difference between the receive signal power and the power in the first path, both acquired from dw1000 chip, to determine whether this is a NLOS measurement, according to the following equation

$$
\left\{\begin{array}{l}
\text { if }\left(P_{r p}-P_{f p}\right) \leq \eta \quad L O S \\
\text { else NLOS }
\end{array}\right.
$$

where

$$
\begin{aligned}
& P_{r p}=\text { the receive signal power } \\
& P_{f p}=\text { the power in the first path } \\
& \eta=\text { the judgment threshold, which is set as } 6.8 \mathrm{~dB} \\
& \quad \text { according to measurement data }
\end{aligned}
$$

The power in the first path and the receive signal power can be calculated based on the value of receive time stamp register in dw1000 chip.

Meanwhile, in order to refine the NLOS identification operation, we define a quantification factor as a likelihood to determine whether this is a NOLS measurement. It can be described as

$$
\lambda=\left\{\begin{array}{l}
0.0, \text { if }\left(P_{f p}-P_{r p}\right) \leq 3.3 \\
k_{1} *\left(P_{f p}-P_{r p}\right)-k_{2}, \text { if } 3.3 \leq\left(P_{f p}-P_{r p}\right) \leq 6.0 \\
1.0, \text { else }
\end{array}\right.
$$

where $\quad k_{1}, k_{2}=$ the system parameters

Based on multiple measurement data, we set the value of $k_{1}$ and $k_{2}$ as 0.421 and 1.283 , respectively.

\subsection{Localization algorithms}

The accuracy of UWB ranging is always affected by the clock drift, antenna delay, dispersed obstacles, objects moving in the scenario, and even the ripple noise of the power supply system. And these errors are usually random and difficult to describe with an accurate mathematical mode. To simplified the ranging model, we model all of these cumulative errors as a Gaussian random process with zero mean and variance $\delta_{i}^{2}$. So, the TOF measurement results between the mobile nodes and the fixed nodes can be described as

$$
d_{i, t}=\sqrt{\left(x_{t}-x_{i}\right)^{2}+\left(y_{t}-y_{i}\right)^{2}}+\alpha_{i, t}
$$

where $\quad d_{i, t}=$ measurement distance between the mobile tag and the $i$-th anchor

$\left(x_{t}, y_{t}\right)=$ location of the mobile tag at time $t$

$\left(x_{i}, y_{i}\right)=$ coordinate of the $i$-th anchor

$\alpha_{i, t}=$ Gaussian noise distributed with zero mean and variance $\delta_{i}^{2}$

Due to the ranging model is modelled as a Gaussian random process. Therefore, an extend Kalman filter (EKF) can be adopted for localization. In this article, we only evaluate the $2 \mathrm{D}$ positioning, so the system state vector $\mathbf{s}_{t}$ can be expressed as

$$
\mathbf{s}_{t}=\left[\begin{array}{ll}
x & y
\end{array}\right]^{T}
$$

And the discrete dynamic system model can be described as

$$
\left\{\begin{array}{l}
\mathbf{s}_{t}=\mathbf{A}_{t} \mathbf{s}_{t-1}+\omega_{t} \\
\mathbf{Z}_{t}=\mathbf{H}_{t} \mathbf{s}_{t}+v_{t}
\end{array}\right.
$$


where

$$
\begin{aligned}
& \mathbf{s}_{t}=\text { the predicted state } \\
& \mathbf{s}_{t-1}=\text { the previous state } \\
& \mathbf{A}_{t}=\mathbf{I}_{2 \times 2} \text {, the state transition matrix } \\
& \omega_{t}=\text { the process noise with covariance matrix } \\
& \text { covariance matrix } Q_{t}=E\left(\omega_{t} \omega_{t}^{T}\right) . \\
& v_{t}=\text { the measurement noise with covariance } \\
& \text { matrix } R_{t}=E\left(v_{t} v_{t}^{T}\right) .
\end{aligned}
$$

Although each available range measurement with short interval can be applied in the EKF localization algorithm. Yet this algorithm mechanism is hardly to converge when serious ranging errors occur. To avoid this problem, all the range measurements obtained from one C-TWR loop are used as the system observations in this paper, which effectively weaken the effect factor of single measurement. Then the observation vector can be constructed as

$$
\mathbf{Z}=\left[\begin{array}{llll}
d_{1} & d_{2} & d_{3} & d_{4}
\end{array}\right]^{T}
$$

where $\quad d_{i}=$ the measurement distance with the $i$-th anchor

The measurement matrix is given by

$$
\mathbf{H}_{t}=\left[\begin{array}{cc}
\Delta x_{1} & \Delta y_{1} \\
\vdots & \vdots \\
\Delta x_{i} & \Delta y_{i}
\end{array}\right]\left[\begin{array}{ccc}
1 & & 1 \\
\frac{d_{1, t+\Delta t}}{d_{i, t+\Delta t}} & \cdots &
\end{array}\right]
$$

where $\quad \mathbf{H}_{t}=$ the linearized measurement matrix at time $t$

$$
\Delta x_{i}=x_{t}-x_{i}, \Delta y_{i}=y_{t}-y_{i}
$$

Meanwhile, the accuracy of ranging is fluctuant due to the body occlusion, it may be unstable especially when the tag and base station are facing the opposite of each other. To improve the robustness of the system, an adaptive EKF (A-EKF) with optimized measurement noise covariance is deployed.

In the A-EKF filter, the measurements noise covariance can be adjusted by mean of residual $\eta$ in real time, represented as

$$
\eta_{i, t}=d_{i, t}-\sqrt{\left(x_{t \mid t}-x_{i}\right)^{2}+\left(y_{t \mid t}-y_{i}\right)^{2}}
$$

where $\quad\left(x_{t \mid t}, y_{t \mid t}\right)=$ coordinates of tag at time $\mathrm{t}$ estimated by $\mathrm{EKF}$

\section{EXPERIMENTS}

In this paper, we use Decawave UWB node as the base stations and mobile tags, Fig. 3 shows the hardware details of the node.

Due to the tag's motility, the TOF ranging results collected from the adjacent anchor nodes are from different time instant. The situation is particularly important and needs special attention for rapid movement systems with the characteristics of fast changes in direction and velocity. The time delay between two range measurements in one integrated C-TWR scheme was set to approximately $0.75 \mathrm{~ms}$ to improve the performance of real-time.

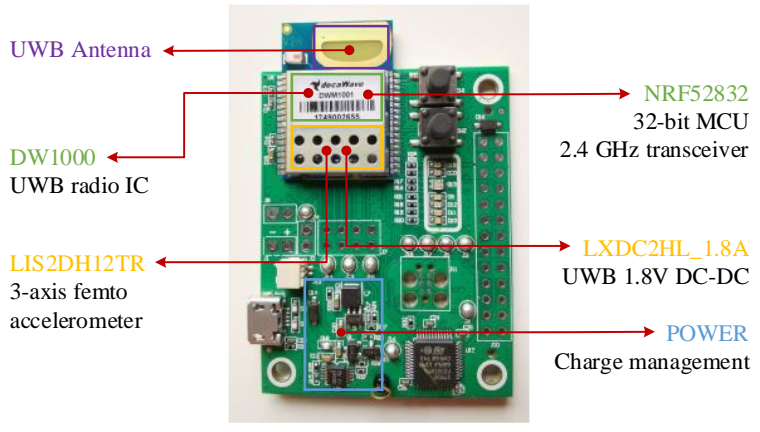

Figure 3. Photo of the Decawave UWB node.

To verify the low delay performance of the node ranging, a specific electro-rotating actuator was designed to provide precise reference for the real speed of the nodes, as shown in Fig. 4. The actuator was driven by a DC motor with programmable speed. We adjusted the linear velocity of the marked nodes to approximately $5 \mathrm{~m} \cdot \mathrm{s}^{-1}$. By optimizing the firmware, a measuring sampling rate of around $256 \mathrm{~Hz}$ can be obtained between one tag and two anchors.

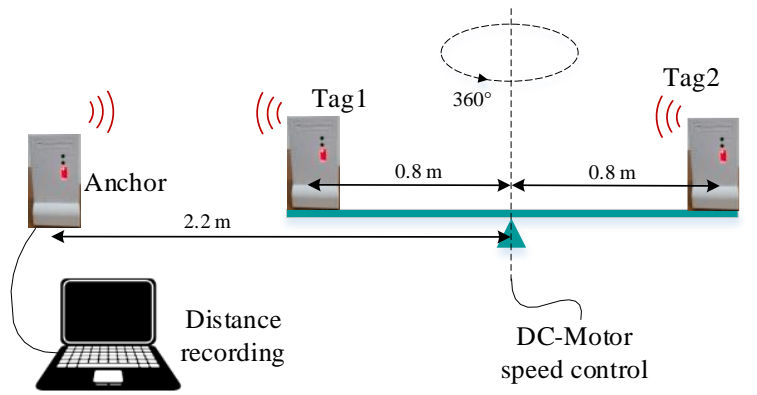

Figure 4. Electro-rotating actuator with programmable speed for evaluating the rapid movement detection.

The test lasted about $20 \mathrm{~s}$. The number of the valid samples points is about 5086 based on the statistics measurement results. The sampling rate can be inferred which is about $254.3 \mathrm{~Hz}$, and the success rate of ranging can be calculated which is better than $99.3 \%$. A detailed view of the ranging measurement results by the Electro-rotating actuator of 5 to 6 seconds is presented in Fig. 5 . The ranging accuracy of both tags is less than $15 \mathrm{~cm}$.

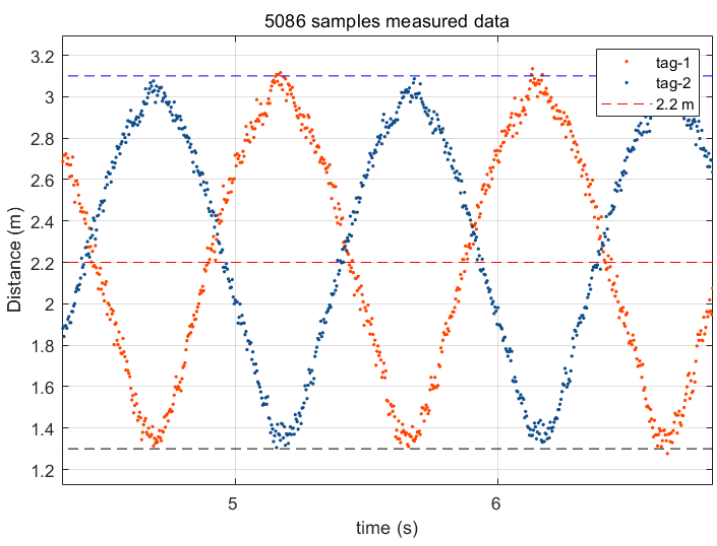

Figure 5. The ranging results of anchor by the Electro-rotating actuator (a detail view of 5 to 6 seconds). 
Furthermore, to evaluate the positioning performance of the system, a test platform consisting of four anchors and four tags was designed to examine the accuracy and robustness of the system. Four anchors were located closely to the corners at a hight of about $2 \mathrm{~m}$ from the ground. Four tags were tightly tied to the pedestrian's hand and leg using a Velcro strap (see Fig. 6). The length and width of the coverage area are $28 \mathrm{~m}$ and 15 $\mathrm{m}$, respectively. The move of pedestrian is characterized by fast changes in direction and velocity followed with a predetermined route, as given in Fig. 6. The coordinates of starting point and ending point is both $(4,2.5)$.

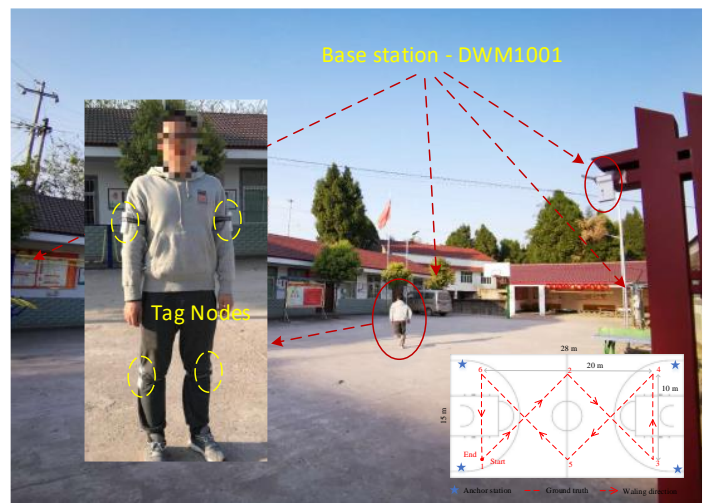

Figure 6. A panoramic view of the base station placement, experimental arena, and the route.

\section{RESULTS}

This section demonstrates the dynamic measurement results of the system for one moving cycle. Fig. 7 indicates a comparison of the reference trajectory with the estimated positions of the pedestrian equipped with four tags.

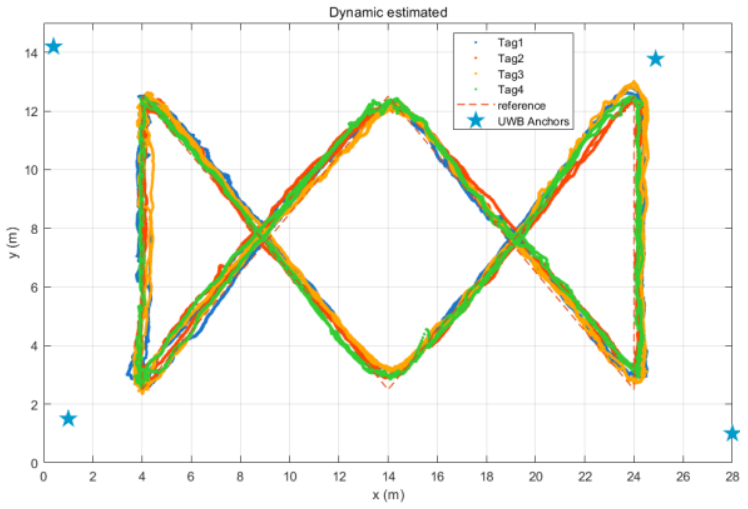

Figure 7. Comparison of estimated positions with reference ones.

It can be seen that the position estimates from all four tags can match the reference trajectory effectively.

Table 1 describes the specific value of the errors.

\begin{tabular}{ccccc}
\hline & Mean $(\mathbf{m})$ & $\mathbf{9 0 \%}(\mathbf{m})$ & $\mathbf{9 5 \%}(\mathbf{m})$ & $\operatorname{Max}(\mathbf{m})$ \\
\hline Tag1 & 0.16 & 0.42 & 0.49 & 0.59 \\
\hline Tag2 & 0.29 & 0.49 & 0.53 & 0.62 \\
\hline Tag3 & 0.28 & 0.39 & 0.43 & 0.56 \\
\hline Tag4 & 0.23 & 0.43 & 0.45 & 0.57 \\
\hline
\end{tabular}

Table 1. Comparison of errors from different tags

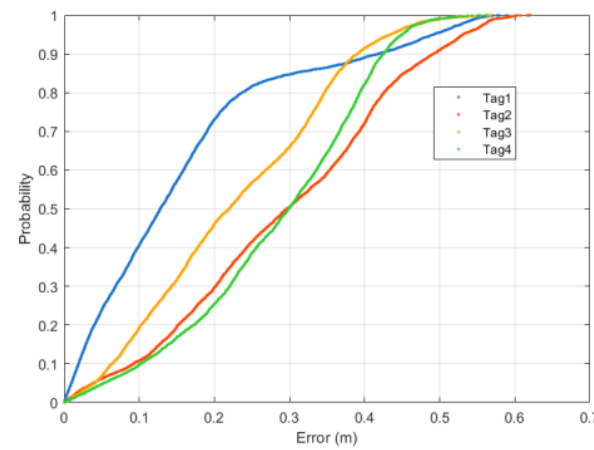

Figure 8. CDF of position estimations

Fig. 8 shows the CDFs of the positioning error, calculated from the Euclidean distance between the estimated positions and the adjacent positions of reference trajectory. From Fig. 8 and Table 1, it is clear that the error of $95 \%$ is less than $0.5 \mathrm{~m}$, the mean error is better than $0.3 \mathrm{~m}$ for most tags. This is mainly benefit from the appropriate algorithms model and adaptive noise model.

As a rule of thumb, the average speed of pedestrian can be calculated based on the definition of speed. Therefore, we can evaluate the average speed according to the coordinate of two consecutive positioning points and the time difference between them. As indicated in Fig. 9, the stable walking maximal speed and minimum speed are about $5 \mathrm{~m} . \mathrm{s}^{-1}$ and $2 \mathrm{~m} . \mathrm{s}^{-1}$, respectively.

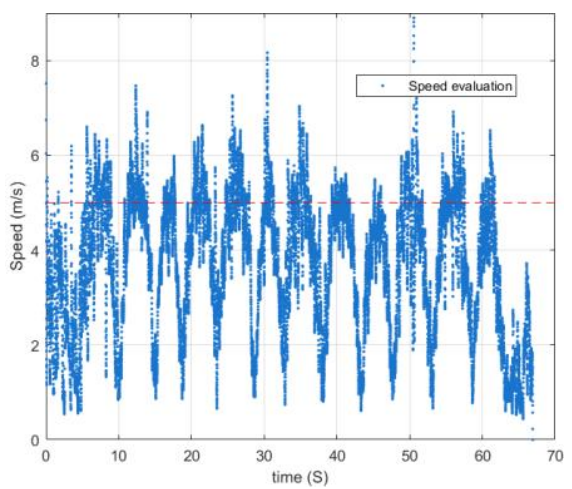

Figure 9. Motion speed.

Finally, Fig. 10 describes the singular value vector of noise covariance $\mathrm{P}$ for EKF metrics. This parameter can be used to measure the robustness of the algorithm, the smaller the value is, the better the performance.

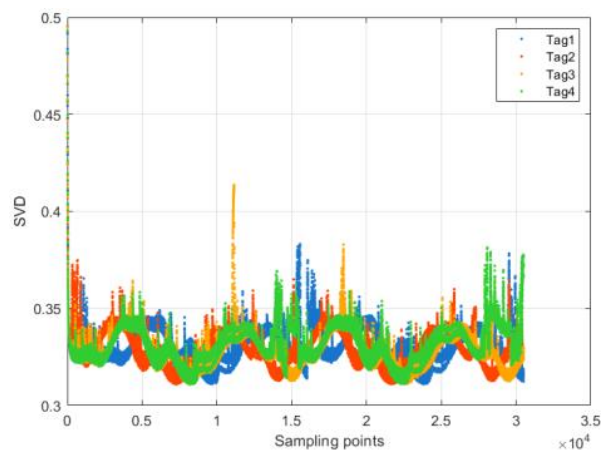

Figure 10. EKF Metrics. 


\section{CONCLUSIONS}

In this paper, a low-delay and robust solution for rapid movement detection using UWB is presented. The measurement results obtained from the experiments show that the system can locate the rapid moving pedestrian with a maximal speed of approximately $5 \mathrm{~m} . \mathrm{s}^{-1}$ in real-time. The positioning accuracy is less than $0.5 \mathrm{~m}$, and the speed of the rapid movement detection is improved. Compared with some tradition motion capture system, this system can provide a better way for sports training in large scale and rapid movement applications with lower cost and better portability.

The research offers implications for further studies in the range of human wearable tags in various situations, especially in sports, such as athletes' positioning and speed statistics during the routine training.

\section{ACKNOWLEDGEMENTS}

This work supported in part by the National Key Research and Development Program of China (grant nos. 2016YFB0502200 and 2016YFB0502201) and the NSFC (grant no. 91638203)

\section{REFERENCES}

Banerjee, S., Hempel, M., Sharif, H., 2017. A review of workspace challenges and wearable solutions in railroads and construction. 201713th International Wireless Communications and Mobile Computing Conference (IWCMC), 91-96.

Ding, R., Qian, Z. H., Wang, X., 2010. UWB positioning system based on joint TOA and DOA estimation. Journal of Electronics and Information Technology, 313-317.

Decawave, 2017. DW1000 User Manual. Decawave Ltd.

Decawave, 2018. Indoor Positioning Systems (IPS) - RTLS Solutions[WWW Document]. URL https://www.decawave.com/ (accessed 5.15.18).

Guolin, S., Jie, C., Wei, G., Liu, K. J. R., 2005. Signal processing techniques in network-aided positioning: a survey of state-of-the-art positioning designs. IEEE Signal Processing Magazine, IEEE, 22, 12-23.

Hammeed, A., Ahmed, H., 2018. Survey on indoor positioning applications based on different technologies. 2018 12th International Conference on Mathematics, Actuarial Science, Computer Science and Statistics (MACS), IEEE.

IEEE Standard for low-rate wireless networks, 2016. IEEE Std 802.15.4-2015 (Revision of IEEE Std 802.15.4-2011), 1-709.

Joo, H., Liu, H., Tan, L., Gui, L., 2015. Panoptic Studio: A massively multiview system for social motion capture. 2015 2015 IEEE International Conference on Computer Vision $(I C C V)$, IEEE.

Koyuncu, H., Yang, S.H, 2011. A survey of indoor positioning and object locating systems. In Proceedings of the International Conference on Innovations in Information Technology, 121128.

Leser, R., Baca, A., Ogris, G, 2011. Local positioning systems in (game) sports. Sensors, 11, 9778-9797.
Nuaimi, K., Kamel, H., 2011. A survey of indoor positioning systems and algorithms. 2011 International Conference on Innovations in Information Technology, IEEE, 185-190.

Pham, D. D., Suh, Y. S., 2016. Pedestrian navigation using Foot-Mounted inertial sensor and LIDAR. Sensors, 16, 120.

Risset, T., Goursaud, C., Brun, X., Marquet, K., Meyer, F., 2018. UWB ranging for rapid movements. 2018 International Conference on Indoor Positioning and Indoor Navigation (IPIN), IEEE, 1-8.

Ridolfi, M., Velde, S., Steendam, H., Poorter, E., 2018. Experimental evaluation of UWB indoor positioning for sport postures. Sensors, 18, 168.

Silva, B., Pang, Z., Akerberg, J., Neander, J., Hancke, G., 2014. Experimental study of UWB-based high precision localization for industrial applications. IEEE International Conference on Ultra-WideBand (ICUWB), IEEE, 280-285.

Vorst, P., Sommer, J., Hoene, C., Schneider, P., Weiss, C., Schairer, T., Rosenstiel, W., Zell, A., Carle, G., 2008. Indoor positioning via three different RF technologies. 2008 4th European Workshop on RFID Systems and Technologies, VDE, $1-10$. 